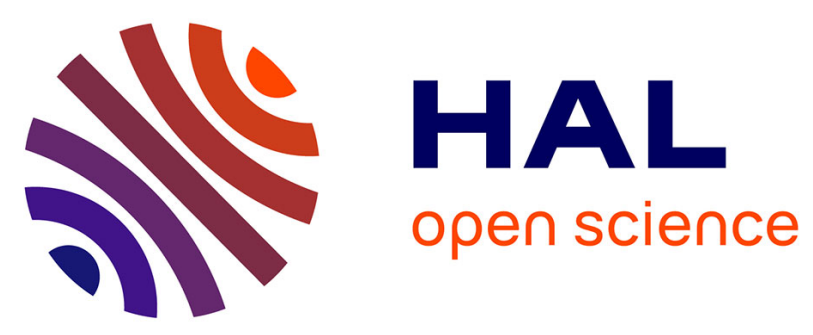

\title{
Value Chain: From iDMU to Shopfloor Documentation of Aeronautical Assemblies
}

Manuel Oliva, Jesús Racero, Domingo Morales-Palma, Carmelo Del Valle, Fernando Mas

\section{- To cite this version:}

Manuel Oliva, Jesús Racero, Domingo Morales-Palma, Carmelo Del Valle, Fernando Mas. Value Chain: From iDMU to Shopfloor Documentation of Aeronautical Assemblies. 14th IFIP International Conference on Product Lifecycle Management (PLM), Jul 2017, Seville, Spain. pp.106-115, 10.1007/978-3-319-72905-3_10. hal-01764176

\section{HAL Id: hal-01764176 \\ https://hal.inria.fr/hal-01764176}

Submitted on 11 Apr 2018

HAL is a multi-disciplinary open access archive for the deposit and dissemination of scientific research documents, whether they are published or not. The documents may come from teaching and research institutions in France or abroad, or from public or private research centers.
L'archive ouverte pluridisciplinaire HAL, est destinée au dépôt et à la diffusion de documents scientifiques de niveau recherche, publiés ou non, émanant des établissements d'enseignement et de recherche français ou étrangers, des laboratoires publics ou privés. 


\title{
Value chain: from iDMU to shopfloor documentation of aeronautical assemblies.
}

\author{
Manuel Oliva ${ }^{1}$, Jesus Racero ${ }^{2}$, Domingo Morales-Palma ${ }^{2}$, Carmelo del Valle ${ }^{2}$, \\ Fernando Mas ${ }^{1}$ \\ ${ }^{1}$ Airbus. Carretera A-8010 km.4 41020 Sevilla \\ ${ }^{2}$ Universidad de Sevilla. \\ manuel.oliva@airbus.com; jre@us.es; dmpalma@us.es; \\ carmelo@us.es; fernando.mas@airbus.com
}

\begin{abstract}
Competition in the aerospace manufacturing companies has led them to continuously improve the efficiency of their processes from the conceptual phase to the start of production and during operation phase, providing services to clients. PLM (Product Lifecycle Management) is an end-to-end business solution which aims to provide an environment of information about the product and related processes available to the whole enterprise throughout the product's lifecycle.

Airbus designs and industrializes aircrafts using Concurrent Engineering methods since decades [2]. The introduction of new PLM methods, procedures and tools, and the need to improve processes efficiency and reduce time-tomarket, led Airbus to pursue the Collaborative Engineering method. Processes efficiency is also impacted by the variety of systems existing within Airbus. Interoperability rises as a solution to eliminate inefficiencies due to information exchange and transformations and it also provides a way to discover and reuse existing information.

The ARIADNE project (Value chain: from iDMU to shopfloor documentation of aeronautical assemblies) was launched to support the industrialization process of an aerostructure by implementing the industrial Digital Mock-Up (iDMU) concept in a Collaborative Engineering framework. Interoperability becomes an important research workpackage in ARIADNE to exploit and reuse the information contained in the iDMU and to create the shop floor documentation. This paper presents the context, the conceptual approach, the methodology adopted and preliminary results of the project.
\end{abstract}

Keywords: PLM, iDMU, interoperability, Collaborative Engineering, assembly

\section{Introduction}

PLM systems integrate all phases in the product development. The full product lifecycle, from the initial idea to the end-of-life, generates a lot of valuable information related to the product [1].

adfa, p. 1, 2011.

(C) Springer-Verlag Berlin Heidelberg 2011 
In aerospace industry, the long lifecycle (about 50 years), the number of parts (over 700.000 as average in a short range aircraft) and the modifications, make the aircraft a high complex product. Such complexity is drawn both from the complexity of the product and from the amount of resources and multidisciplinary work teams involved.

A complexity of multidisciplinary is found during the interaction between functional and industrial designers which brings inefficiencies in developing time, errors, etc. Research studies propose the necessity to evolve from the concurrent way of working to a more efficient one with the objective to deliver faster, better and cheaper products [2], [3], [4]. One proposal to comply with such challenge is the Collaborative Engineering concept [5], [6].

Collaborative Engineering involves a lot of changes in terms of organization, teams, relationships, skills, methods, procedures, standards, processes, tools, and interfaces: it is a business transformation process. The main deliverable of a collaborative team is the iDMU [7]. The iDMU concept is the approach defined by Airbus to facilitate the integration of the aircraft development on a common platform throughout all their service life. An iDMU gathers all the product, processes and resources data, both geometrical and technological to model a virtual assembly line. An iDMU provides a single environment, in which the assembly line industrial design is defined and validated.

To cover the bridge between the complexity of product information and the different PLM software tools to manage it, interoperability has raised as a must nowadays to improve the use of existing data stored in different formats and systems [8]. Interoperability foundation is the Model Base Engineering (MBE), as a starting point for organizing a formal way of communicating and building knowledge [9] from data and information.

The development of solutions, to facilitate the implementation of both the concurrent engineering and the Collaborative Engineering in the aerospace industry, was the objective of some projects since the end of the 1990's decade. Two of the most relevant ones are the European projects ENHANCE [10], [11] and VIVACE [12].

In the last decade, different research projects have been conducted for a complete integration of the iDMU and all the elements in the different stages of the life cycle (from design to manufacturing). The CALIPSOneo project [13] was launched by Airbus to promote the Collaborative Engineering. It implements the iDMU as a way to help in making the functional and the industrial designs evolving jointly and collaboratively. The project synchronizes, integrates and configures different software applications that promote the harmonization of common set of PLM and CAD tools.

EOLO (Factories of the future. Industrial development) project was developed as an initiative to achieve a better integration between information created in the industrialization phases and the information created in the operation and maintenance phases.

The ARIADNE project emerges as an evolution of both, CALIPSOneo and EOLO projects, which incorporates the integrated management of the iDMU life cycle (product, processes and resources), the Collaborative Engineering and interoperability between software systems (independent vendor). These characteristics will provide an improvement of data integration, knowledge base and quality of the final product. 


\section{ARIADNE project}

\subsection{ARIADNE project organization}

ARIADNE is organized around three work packages: MINOS, HELIOS and ORION. The relationship between work packages is shown in Figure 1.

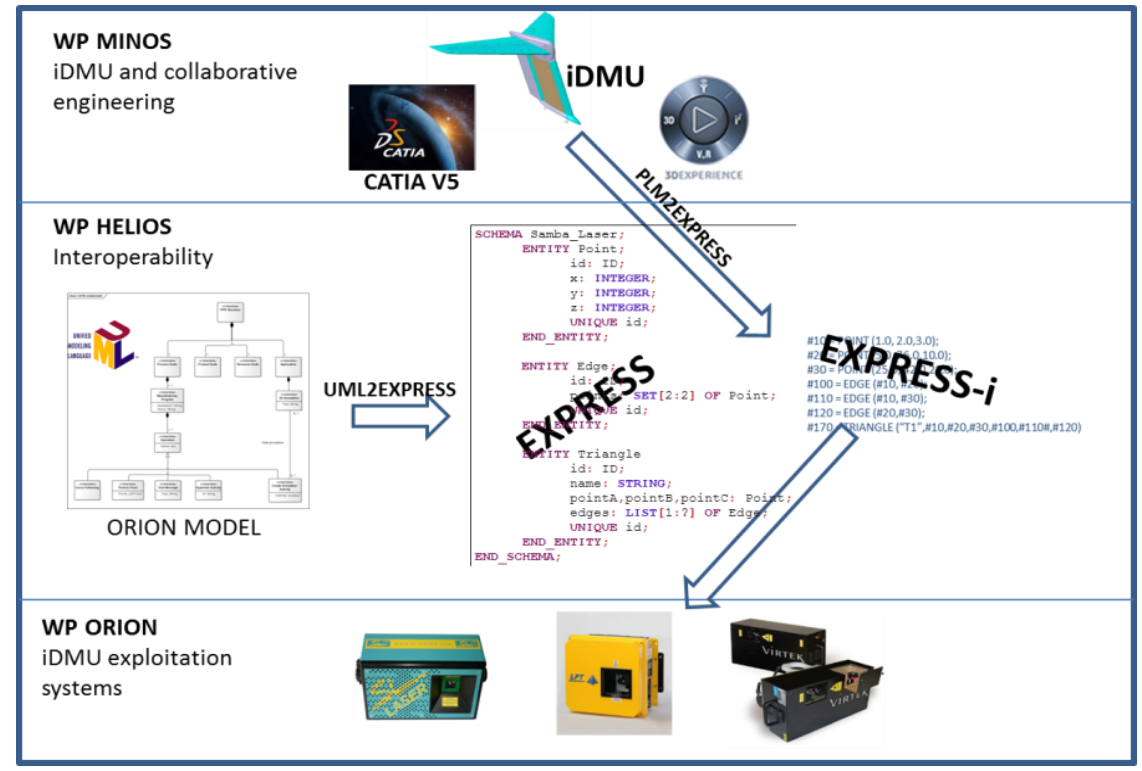

Figure 1. ARIADNE project organization

- MINOS (Competitive new PLM systems interoperability scenario). Main target in this work package is to analyze the functionalities provided by a commercial PLM, 3DExperience by Dassault Systémes [18], to build an iDMU and support Collaborative Engineering. A second target is to study interoperability between CATIA v5 by Dassault Systémes [18], the PLM platform currently running in most of the aerospace companies, and 3DExperience. An analysis of the 3DExperience platform is been performed in ARIADNE with the objective of checking the main functionalities needed for the industrial design, that represents an improvement over CATIA v5. It is not an exhaustive analysis of all functionalities of 3DExperience, but it is a study of the characteristics provided by 3DExperience that covers the main requirements of manufacturing engineering activities for the industrialization of an aerospace assembly product.

- HELIOS (New shopfloor assembly documentation models). HELIOS proposes research on a solution to extract information from an iDMU independently of the software provider. The conceptual solution is based on developing the models and transformations needed to explode the iDMU for any other external system. Currently, any system that needs to exploit the iDMU would have to develop its own 
interfaces. In case the iDMU is migrated to a different PLM, those interfaces must be changed also. To help with those inefficiencies and to be independent from any existing PLM, HELIOS will generate a standardized software code (EXPRESS-i) that any external system can use to communicate and obtain the required information from the iDMU.

- ORION (Laser authoring shop floor documentation). ORION aims to develop a system to exploit assembly process information contained in the iDMU with Augmented Reality (AR) technics using laser projection technology. This system will get any data from the iDMU needed for the assembly, verification or maintenance process. ORION is based on the SAMBAlaser project [14], an 'AR by laser' technology developed by Airbus. ORION will analyze new ways for laser programming besides numerical control and will provide a 3D simulation tool. Also it will propose a data model to integrate the iDMU with the AR laser system and to facilitate the laser programming and execution.

\subsection{ARIADNE project functional architecture}

ARIADNE architecture is a consequence of the conclusions and the proposed future work in CALIPSOneo project in 2013. CALIPSOneo architecture for a collaborative environment was CATIA v5 in conjunction with DPE (DELMIA Process Engineering) to hold the process definition in a database (also called Manufacturing Hub by Dassault Systèmes). The architecture in CALIPSOneo, although still in production in Airbus and available in the market, is not and architecture ready to support the requirements from Industry 4.0 and is quite out of phase in technology to connect or communicate with today's technology.

To develop MINOS, the decision on the tool to support it was 3DExperience a natural evolution of CATIA v5. Data used in MINOS, the Airbus military transport aircraft A400M Empennage shown in Figure 3a, are in CATIA v5 format. To keep the 3DExperience infrastructure simple, and thanks to the relative low volume of data of the A400M empennage, a single virtual machine with all the required servers were deployed for the project.

For the interoperability between CATIA v5 and 3DExperience, CATIA v5 the input data are stored in file based folders containing the geometry in CATPart and the product structure in CATProduct as Figure 5. FBDI (File Based Data Import) is the process provided by Dassault Systémes that reads and or imports information (geometry and product structure) into 3DExperience. The option 'Import as Native', selected in FBDI will read the CATIA v5 as a reference, meaning, creating a 3D representation in 3DExperience as in CATIA v5, but will not allow it to be modified. Resources and assembly processes will be designed in 3DExperience based on the product (in CATIA v5) previously imported. For the interoperability analysis, the wing tip of the Airbus C295 (a medium range military transport aircraft) was chosen.

Developments in HELIOS and ORION will be based also on CATIA v5 data availability. 
ARIADNE pretends to use only off-the-shelf functionalities offered natively by 3DExperience with no additional development.

\section{$3 \quad$ Implementation and results}

Collaborative Engineering, interoperability and iDMU exploitation are the targets in the different work packages of ARIADNE. The implementation and results are described in this section.

\subsection{Collaborative Engineering}

Collaborative Engineering requires an integrated 3D environment where functional and industrial engineers can work together influencing each other. The main driver for the Collaborative Engineering method is the construction of the iDMU. ARIADNE is focused in the collaboration between the functional design and industrial design teams. ARIADNE will check if 3DExperience provides such environment to build the iDMU where Collaborative Engineering can be accomplish.

To analyze 3DExperience collaborative environment a few use cases were defined and tested with the Airbus A400M empennage product represented in Figure 2a.

One of the bases to integrate the information in a PLM is to be able to hold the different ways or views (As-Design, As-Planned, As-Prepared) [15], shown in Figure 2b, for defining the product in Airbus. Keeping these views connected is basic to the Collaborative Engineering [7]. In the work performed it was possible to build the AsDesign view. Following, the As-Planned view was built from the As-Designed view while sharing the same 3D geometry for each of the structures. This is represented in Figure $3 b$.

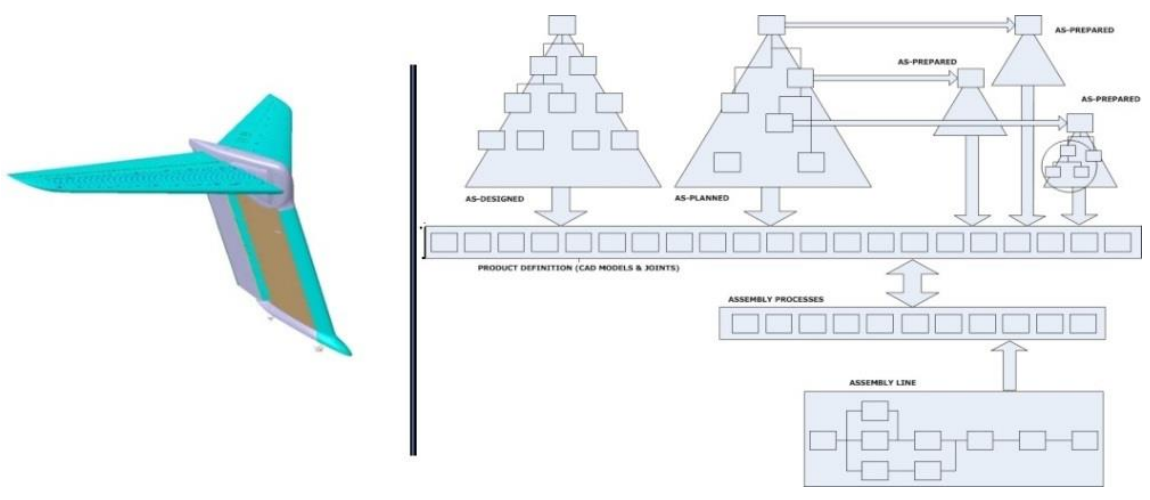

Figure 2. a) Empennage A400M. b) Airbus product views

A set of additional functionalities were performed in the As-Planned view such as the possibility of navigating through the structure as well as in the As-Design or filtering of product nodes in the product tree. Reconciliation in 3DExperience has proven to be an important functionality to assure a fully connection As-Design and As- 
Planned. This functionality indicates in the tree with a color code the issues found when comparing both structures.

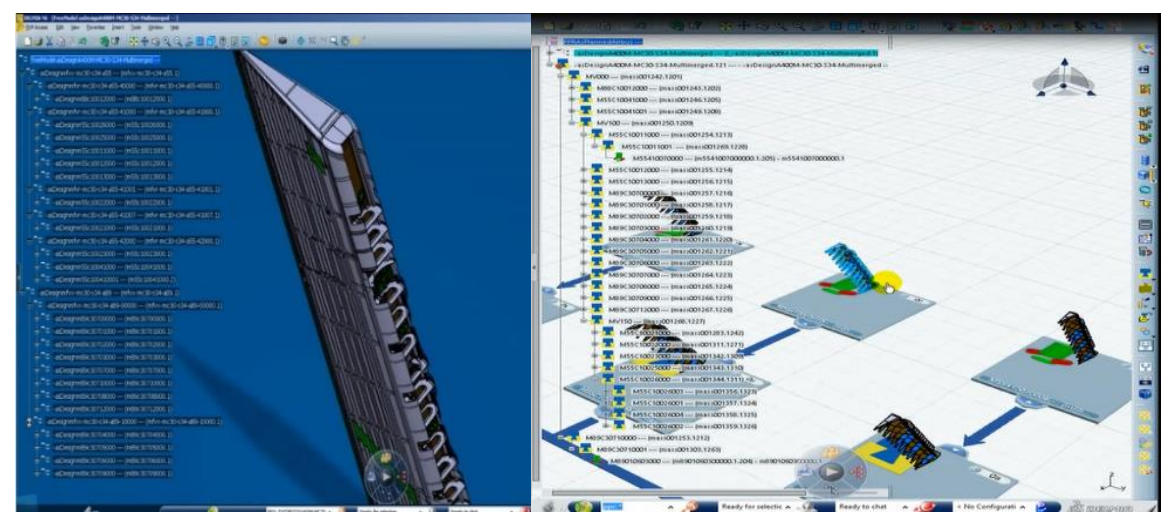

Figure 3. a) As-Design view. b) As-Planned view

\subsection{Building the iDMU.}

The third structure created in ARIADNE, which is the main one used for the industrialization of a product, is the As-Prepared. This structure is also a product structure rearranged as a result of the different assembly processes needed to build the product. The As-Prepared tree organization shown in Figure $4 \mathrm{a}$ is a consequence of the network of assembly processes. To build such network, precedence between assembly processes and operations must be assigned as in Figure 4b. Also tools like Gantt representation in Figure $4 \mathrm{c}$ helps deciding the precedence based on constrains (resources and times). Additional functionality for balancing constrains is too basic in 3DExperience for Airbus product complexity. Optimization tool is not offered by 3DExperience. Additional development would be needed to cover these last two functionalities [16].

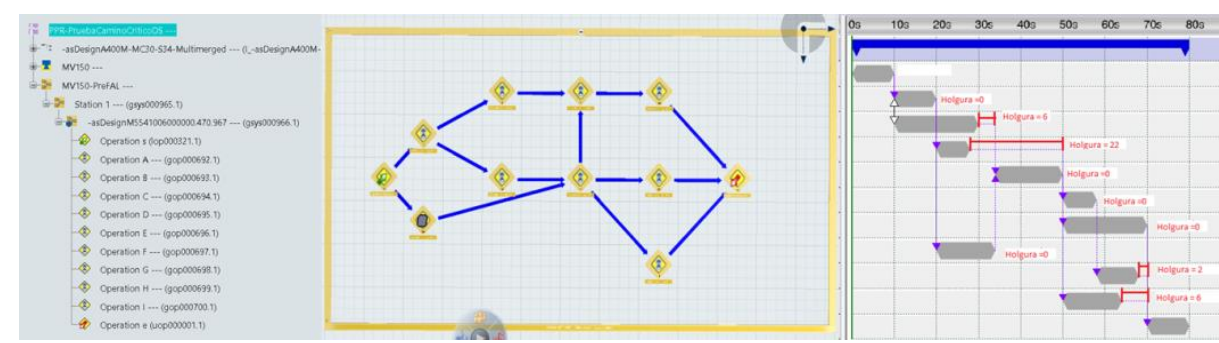

Figure 4. a) As-Prepared. b) Precedence between operations. c) Operations Gantt chart

The iDMU was built by assigning product and resources to each operation together with the precedence. With such information in the iDMU, the use case design in context was performed. The design of an assembly process or a resource requires the representation of the product and the industrial environment based on the operations 
previously performed. This context was possible to be calculated and represented in 3DExperience.

The reconciliation between As-Planned and As-Prepared was tested to make sure that every product was assigned to a process. This functionality is also shown in the tree process structure with color code nodes.

ARIADNE analyzed the capabilities to check how functional designers and industrial designers could carry out their activities influencing each other. For this, a mechanism to follow the evolution of the maturity states of the product, process and resources was proposed [19]. This mechanism is intended to foster an interaction between both design areas.

\subsection{Interoperability CATIA v5 and 3DExperience.}

Recent developed aircrafts (A380, A350 and A400M) in Airbus have been designed in CATIA v5. Migration of a complete product design of an aircraft requires a high effort in resources and cost. Finding a solution where the product design can be kept in CATIA v5 while downstream product lifecycle uses a more adequate environment to cover their activities becomes a target for MINOS work package.

MINOS analyzed the degree of interoperability between 3DExperience and CATIA v5. Interoperability in this use case is understood as the set of required characteristics to develop the industrialization activities performed by manufacturing engineering in 3DExperience without affecting the product design activities (functional design) of the design office performed in CATIA v5.

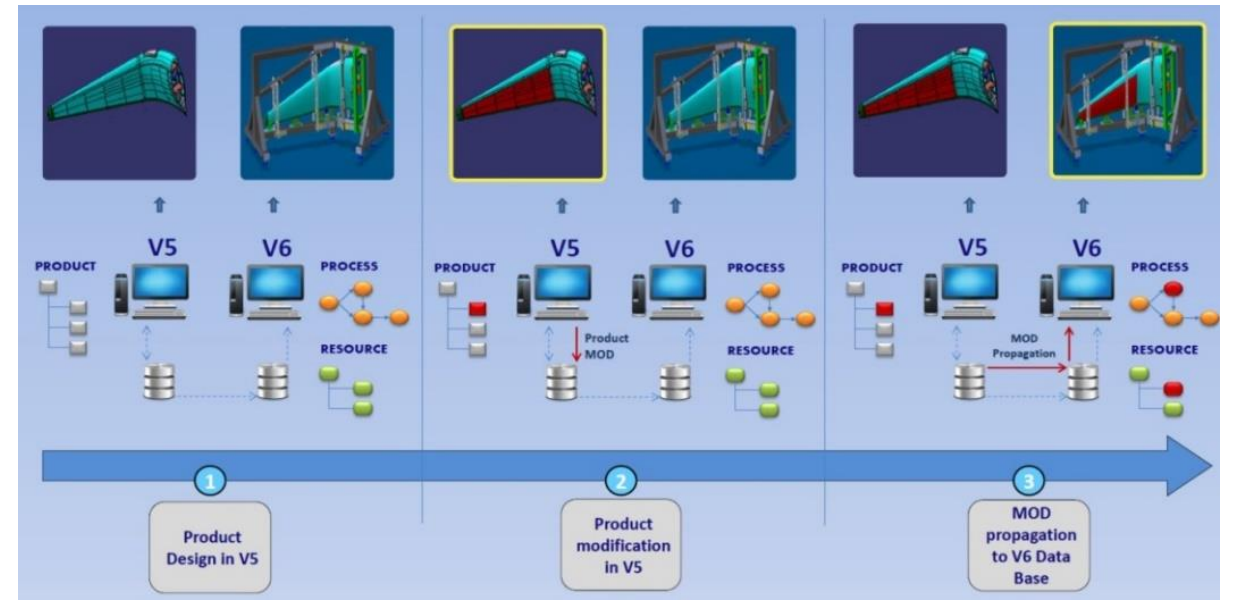

Figure 5. Interoperability CATIA v5 (functional design) and 3DExperience (industrial design)

Initially a reading of product design (product structure and product design) in CATIA v5 was carried out in 3DExperience, step 1 in Figure 5. Checking the result of such work in 3DExperience demonstrated a successful import of the information for the product structure and for the $3 \mathrm{D}$ geometry. Following, a modification was intro- 
duced in the CATIA v5 product, step 2 in Figure 5. FBDI process did detect such change of the product and propagated it in 3DExperience, step 3 in Figure 5. Also 3DExperience sent a warning to update the product structure with the modified product. An impact on the process and resources related to the modified product was performed based on functionalities provided by 3DExperience.

\subsection{Interoperability and iDMU exploitation.}

Due to the increasing added value that the iDMU provides, it becomes an important asset to a company. Once assembly processes are designed and stored in the iDMU, information to production lines to perform the tasks can be extracted with an automatic application system.

As the current production environment in Airbus is CATIA v5, extracting information from the iDMU is constrained to such environment. HELIOS has developed an interoperable framework based in a set of transformations to exploit the iDMU independently from the PLM vendor and STEP is the tool selected. The use case HELIOS is based on is the ORION UML (Universal Model Language) model. The ORION UML model is transformed (UML2EXPRESS) into a schema defined in a standard language such as EXPRESS [17]. The schema is the input to any PLM (CATIA v5 or 3DExperience) to extract the information from the iDMU with a second set of transformations (PLM2EXPRESS). This last transformation will generate the instantiated code (EXPRESS-i) with the required information. This standardized code will be the same input to the different laser vendors.

Currently, in Airbus, the SAMBAlaser [14] is in production for projection of work instructions. To enhance the SAMBAlaser functionalities, ORION work package has developed an integrated user interface with the laser system control, optimized the quantity of information to project without flickering and built a simulation tool to check the capabilities of projecting within an industrial environment without occlusion.

\section{Conclusions}

Main conclusion is the successful proof of concept of the existing PLM technology in an industrial environment.

As mentioned, first test of interoperability CATIA v5 and 3DExperience was successfully. As preliminary conclusions it would be possible for industrialization engineers to work in a more advanced environment, 3dExperience, while functional designers can keep working in CATIA v5. Additional in-depth use cases (annotations, kinematics, and tolerances) need to be performed to check the degree of interoperability.

The introduction of HELIOS as the framework that 'separates' or make any iDMU exploitation system independent of the PLM that support it, is an important step for interoperability between different PLM systems and vendor independency and also enhances the necessity to have a model based definition for the iDMU. Thus, once 
3DExperience becomes the production environment in Airbus, ORION will not need to be modified. HELIOS will be able to support any other iDMU exploitation system just by expanding the UML model.

The three views interconnected (As-Design, As-Planned, As-Prepared) together with the capability of creating a network of processes and operations have proven to build an iDMU to support the collaboration engineering and the facilitation of the interaction between functional an industrial engineers.

3DExperience has demonstrated to provide an interoperable collaborative 3D PLM environment to the industrialization of aeronautical assemblies. However an enterprise organizational model must be put in place to bring together functional and industrial engineering as one team with the iDMU as the unique deliverable.

Since ARIADNE is a proof of concept, no direct estimates on cost, time or other benefits are measured. However, based on previous experiences, significant benefits (time, costs, and reduction of errors) are expected after the deployment phase.

\section{$5 \quad$ Future work}

The current status of ARIADNE project suggests some improvements and future work after the proof of concept of the technology.

ARIADNE project has tested some basic 3DExperience capabilities. The need to explore the 3DExperience capabilities to support the industrialization of an aircraft requires launching additional industrial use cases to cover industrialization activities.

ARIADNE project avoids developing IT interfaces. Connection and interfaces from other tools that provide solutions not fully covered by 3DExperience such as line or station balancing and optimization might need to be analyzed.

ARIADNE objective was not to test computing performances. Performing stress test with high volume of data (metadata, 3D geometry) is another important point to study, mainly for the aerospace industry.

\section{Acknowledgments}

Authors wish to thanks to Andres Soto, Gonzalo Monguió and Andres Padillo for their contributions. ARIADNE is partially funded by CTA (Corporación Tecnológica Andaluza) with support from the Regional and National Government.

\section{$7 \quad$ References}

[1] Ameri, F. and Deba D. 'Product lifecycle management: closing the knowledge loops'. Computer-Aided Design and Applications (2005): 577-590. 
[2] Pardessus, T. 'Concurrent Engineering Development and Practices for aircraft design at Airbus', Proc. of the $24^{\text {th }}$ Intl. Congress of the Aeronautical Sciences (ISCAS 2004), 2004.

[3] Haas, R.; Sinha, M. 'Concurrent engineering at Airbus - a case study'. Int. Journal of Manufacturing Technology and Management, 6:3/4, (2004), 241-253.

[4] F. Mas, J. Ríos, J. L. Menéndez, J. C. Hernández, A. Vizán; “Concurrent conceptual design of aero-structure assembly lines", Proceedings of the 14th Intl. Conf. on Concurrent Enterprising (ICE). Lisbon, 2008.

[5] S.C-Y. Lu, W. Elmaraghy, G. Schuh, R. Wilhelm. "A scientific foundation of Collaborative Engineering”, CIRP Annals - Manufacturing Technology, Volume 56, Issue 2, 2007, pp. 605-634.

[6] Mas Morate, Fernando, et al. "Collaborative Engineering Paradigm Applied to the Aerospace Industry." Proceedings of 10th IFIP WG 5.1 International Conference, PLM 2013, Nantes, France 675-684.

[7] Mas, F. et al. 'iDMU as the Collaborative Engineering engine: Research experiences in Airbus', 2014 International ICE Conference on. IEEE, 2014.

[8] Penciuc, Diana, et al. "Towards a PLM interoperability for a collaborative design support system." Procedia CIRP 25 (2014): 369-376.

[9] Liao, Yongxin, et al. "Semantic annotations for semantic interoperability in a product lifecycle management context." International Journal of Production Research (2016): 5534-5553.

[10] H. Braudel, M. Nicot, J.-C. Dunyach. Overall Presentation of the ENHANCE Project. Air \& Space Europe, 3:3-4, (2001), 49-52.

[11] VIVACE Project: http://ec.europa.eu/research/transport/projects/items /vivace_en.htm (last access March 2017).

[12] T. Nguyen Van, F. Féru, et al. "Engineering Data Management for extended enterprise" - Context of the European VIVACE Project. Intl. Conf. on Product Lifecycle Management, Bangalore, India (2006).

[13] Mas, F., et al. "PLM based approach to the industrialization of aeronautical assemblies." Procedia Engineering (2015): 1045-1052.

[14] Serván, J., et al. "Augmented Reality Using Laser Projection for the Airbus A400M Wing Assembly" Proceedings of the 29th Int. Manufacturing Conference. 2011.

[15] Mas, Fernando, et al. "Proposal for the conceptual design of aeronautical final assembly lines based on the Industrial Digital Mock-Up concept." IFIP Int. Conference on Product Lifecycle Management. Springer Berlin Heidelberg, 2013.

[16] Rios, J.; Mas, F. and Menéndez, J.L. 'A review of the A400m final assembly line balancing methodology.' AIP Conference Proceedings. Vol. 1431. No. 1. AIP, 2012.

[17] ISO 10303-21:1994 (1994) 'Industrial automation systems and integration Product data representation and exchange'. International Organization for Standardization.

[18] https://www.3ds.com/ (last access, March 2017).

[19] Morales-Palma, D.; Eguía, I. 'Managing maturity states in a collaborative platform for the iDMU of aeronautical assembly lines'. Submitted to IFIP 14th .International Conference on Product Lifecycle Management, 9-12 July 2007, Seville, Spain 